\title{
L'affaire Marie Besnard : une querelle d'experts qui s'achève par un acquittement
}

\section{The Marie Besnard case: an expert quarrel which ends with a verdict of not guilty}

Jean-Pierre ANGER ${ }^{(1)}$, Jean-Pierre GOULLÉ( ${ }^{(2)}$

(1) Laboratoire de Toxicologie Pharmaceutique, Université de Rennes 1 - 35043 Rennes Cedex (2) Laboratoire de Pharmacocinétique et de Toxicologie Cliniques, Groupe Hospitalier du Havre,

BP 24 - 76083 Le Havre Cedex

Tirés à part : Jean-Pierre ANGER, 10, allée de la Rivière - 35760 Saint Grégoire

(Reçu le 21 juin 2006 ; accepté après modifications le 11 octobre 2006)

\begin{abstract}
RÉSUMÉ
L'histoire commence en octobre 1947 lorsque Léon Besnard, bourgeois de Loudun (Vienne, France) qui tient un commerce de cordes, tombe malade. Le médecin diagnostique une crise de foie. Une analyse sanguine révèle un excès d'urée. Il meurt le 25 octobre. Très vite la rumeur publique accuse sa femme Marie Besnard d'avoir mis quelque chose dans la soupe de son mari. De l'arsenic! C'est elle aussi, dit toujours la rumeur, qui aurait empoisonné onze autres personnes de sa famille dont sa mère. En 1949, saisie d'un zèle accusatoire, la justice inculpe cette quinquagénaire qu'un psychiatre avait dans ses conclusions décrite comme « anormalement normale»! Ce travail se propose de rappeler les faits de ce feuilleton judiciaire qui passionna la France durant plus d'une décennie, de décrire les analyses toxicologiques menées et l'important travail de la défense qui a fini par confondre des experts renommés et contribué ainsi à créer le doute chez les jurés qui ont finalement décidé d'acquitter l'inculpée en 1961 après trois procès, treize exhumations, de multiples interrogatoires et maints rapports d'expertise.
\end{abstract}

\section{MOTS-CLÉS}

Affaire Marie Besnard ; arsenic ; empoisonnement.

\section{SUMMARY}

The story begins in October 1947 when Leon Besnard, a bourgeois living in the city of Loudun (Vienne, France) who had a rope shop, fell ill. The doctor diagnosed a liver attack. The blood analysis reveals an hyper uraemia. Leon died on October 25th. Immediately, public rumour accused his wife Marie Besnard who was suspected of having added something to her husband's soup, probably arsenic? The rumour also was that Marie might have poisoned eleven other members from Leon's family including his mother. In 1949, the prosecutor was seized by an accusatory zeal and took this fifty-year-old woman before the courts. The aim of this paper was to recall these events, which fascinated France for more than a decade, and then to describe the toxicological analysis carried out and the important work of the defence, which had astounded the court at the end of the proceedings using renowned experts. This contributed in leaving the members of the jury in doubt. Finally, Marie Besnard was acquitted in 1961 after three trials, thirteen exhumations, multiple interrogations and expert's reports.

\section{KEY-WORDS}

The Marie Besnard case ; arsenic ; poisoning. 


\section{Une vie laborieuse semée d'embûches (1).}

Marie Joséphine Philippine Davaillaud est la fille de Pierre et Marie-Louise, née Antigny, travailleurs acharnés de la terre qui vivent à la ferme des Liboureaux de la commune de Saint Pierre de Maillé (Vienne). Elle naît le 15 août 1896. Ses parents pensent que l'instruction est le meilleur moyen pour réussir dans la vie. Marie est inscrite à l'école des sœurs puis, à la fermeture du couvent, à l'école publique de Saint Pierre de Maillé. À 12 ans, une fièvre typhoïde la retient loin de la classe ; elle ne peut pas passer son certificat d'études et met fin à sa scolarité. Adolescente, elle participe aux travaux de la ferme et jusqu'à l'âge de 20 ans, elle s'occupe avec ses parents des labours, des moissons et des animaux. Passée la première guerre mondiale, Marie épouse en premières noces son cousin Auguste Antigny, le 6 avril 1920. Elle est âgée de 24 ans. Le jeune couple habite la ferme des Liboureaux avec les parents. Malheureusement, après quatre années de travail, Auguste est atteint de pleurésie. Il survit et trouve une place de concierge, de gardien et de jardinier dans un château voisin. Brusquement, fin 1926 il est obligé de s'aliter, atteint d'hémoptysie. Durant six mois, il s'achemine lentement vers la mort qui survient le $1^{\text {er }}$ juillet 1927 ; il laisse une jeune veuve de 31 ans. Marie ne comprend pas ce qui lui arrive et sombre dans un état dépressif. Depuis son veuvage, elle entretient une correspondance suivie avec une cousine de Loudun, Pascaline Antigny qui l'invite. À sa table, Marie va rencontrer un voisin, Léon Besnard 35 ans qui tient un magasin de cordes et qui loge ses chevaux dans le hangar de sa cousine. La rencontre ne laisse en rien présager d'autre qu'un échange de politesse et Marie ne reste que peu de temps à Loudun. De retour aux Liboureaux, avec le travail quotidien, il n'y a pas de place pour les souvenirs. En janvier 1929, Marie surprise reçoit une lettre de Léon Besnard qui souhaite la revoir. Marie n'y est pas prête. Après deux demandes en mariage, Léon voit sa demande aboutir. La cérémonie a lieu le 12 août 1929 à Saint Pierre de Maillé. Marie à 33 ans connaît la joie d'une seconde lune de miel. Après un voyage de noces de quatre jours au Pays Basque, les époux regagnent le domicile de Léon à Loudun. Marie est un peu dépaysée à la ville. La place du marché de Loudun (5000 habitants environ) est propice au colportage des ragots de la cité et personne n'est épargné, pas plus le ménage Besnard que les autres ! Léon et Marie forment un couple réputé fortuné, mais les mauvaises langues sont jalouses de leur situation. De nombreux décès familiaux surviennent au début de la seconde guerre mondiale et ces disparitions les conduisent à déménager. Ainsi dès 1941, ils résident dans la maison des beaux-parents où Marie accueille sa mère et une amie Blanche Rivet. Marie visite tous les dimanches ses chers disparus au cimetière et se présente comme une personne très dévote : elle assiste à tous les offices. Le travail ne manque pas après la guerre et le couple Besnard accueille chez lui un jeune prisonnier allemand "Ady » que les commères de Loudun font vite passer pour l'amant de Marie qui sera traitée de « collabo ».

\section{L'événement qui va tout déclencher : la mort de Léon Besnard.}

Durant l'hiver 1946 Léon est victime d'une forte grippe et reste trois mois alité. Marie supporte le surcroît de travail. À l'entrée de l'automne suivant le couple Besnard, après une intense saison d'été à travailler aux champs, décide d'aller passer quelques jours à la ferme des Liboureaux, pour s'assurer que tout va bien. Léon y travaille beaucoup s'occupant des réparations courantes pour laisser la ferme en bon état pendant les mois de l'hiver. Le 16 octobre 1947, avant de repartir à Loudun, Marie a préparé le déjeuner : soupe, œufs au plat, haricots, viande, vin et café. Après le repas, Léon part regonfler les pneus de la voiture avant de prendre la route, mais cet effort semble-il l'a fait vomir. Il ramène pourtant la famille à Loudun, effectue quelques rangements et avant de passer à table pour le dîner, là l'envie de vomir le reprend. Il monte se coucher et s'endort rapidement. Au réveil, il présente les mêmes symptômes que la veille. Marie ne se sent pas bien non plus. Le médecin traitant conclut à une simple crise de foie et leur conseille la diète. Marie se remet rapidement, mais les symptômes persistent chez Léon. Un autre médecin appelé à son chevet fait pratiquer une analyse de sang qui révèle une urée sanguine modérément augmentée à $1,40 \mathrm{~g} / \mathrm{L}$. Léon ne s'en remettra pas et 9 jours plus tard, le 25 octobre 1947 il rend son dernier soupir mettant un terme à 18 ans de bonheur auprès de Marie. Une fois la douleur de l'enterrement passée, Marie se retrouve seule avec sa mère et Ady pour qu'il s'occupe des affaires agricoles dont il a l'habitude. On vient à peine d'enterrer Léon seulement quelques jours avant la Toussaint, que Louise Pintou, la postière, rend visite à ses amis, les frères Massip pour leur faire part de confidences que lui aurait fait Léon qui, selon lui aurait été empoisonné par son épouse. Auguste Massip consulte un dictionnaire médical et à la lecture du texte sur l'arsenic, il demande à Louise si les symptômes de l'intoxication correspondent à ceux 
constatés sur Léon. Elle le lui confirme et Auguste Massip écrit au Procureur de la République de Poitiers, le 4 novembre 1947. Cette lettre fera partie des pièces maîtresses du dossier d'instruction. Une enquête discrète est menée par la gendarmerie, mais elle aboutit à classer l'affaire sans suite. Cependant la rumeur s'amplifie dans Loudun : les jours de marché, dans les cafés, à la poste, les Loudunais semblent avoir eu connaissance du contenu de la lettre d'Auguste Massip. Des regards soupçonneux n'en finissent pas de se poser sur elle.

Un an plus tard, le 17 octobre 1948, un incendie se produit au château de Montpensier, demeure des frères Massip. Ils y perdent tout. Auguste entre dans une rage folle et accuse Marie Besnard d'être l'incendiaire par vengeance en raison de la fameuse lettre qu'il a écrite au Procureur. Quelques jours plus tard les auteurs de l'incendie sont retrouvés : il s'agit en fait de deux enfants des domestiques employés chez les Massip qui jouaient avec des allumettes : ils ont malencontreusement mis le feu à des fanes de haricots et l'incendie s'est propagé très rapidement. Quelques mois plus tard, Marie a l'immense douleur de perdre sa mère, le 14 janvier 1949, des suites d'une mauvaise grippe. Auguste Massip rancunier, reste persuadé que c'est Marie qui a échafaudé la mise en scène de l'incendie et il alerte à nouveau les enquêteurs. Le commissaire Nocquet et l'inspecteur Normand, jeunes officiers de police judiciaire ainsi que le juge d'instruction de Loudun Pierre Roger, dont ils dépendent, tous très ambitieux pour leur carrière, mènent l'enquête, aidés en cela par Auguste Massip. Ce dernier dresse la liste des douze décès survenus dans la famille de Marie Besnard . De plus, chacun d'eux s'accompagne de legs en faveur de Marie ou d'héritages tombés par succession dans son patrimoine.

Peu après survient un cambriolage suspect chez Madame Pintou qui avoue au cours de l'interrogatoire, la confidence que lui aurait faite Léon sur son lit de mort. L'exhumation du corps de Léon est demandée par le Procureur. Elle a lieu le 11 mai 1949. Les prélèvements effectués par le docteur Guillou sont adressés au docteur Georges Béroud du laboratoire de police scientifique de Marseille, expert assermenté près la Cour d'appel d'Aix-en-Provence.

\section{L'arrestation et l'inculpa- tion de Marie Besnard}

Le 21 juillet 1949, Marie est arrêtée chez elle à 9 heures du matin et conduite au commissariat de Poitiers où elle est de nouveau interrogée. Elle ne cesse de répéter que son mari n'est pas mort empoisonné par qui que ce soit. Elle apprend cependant les résultats positifs de l'expertise du docteur Béroud qui, utilisant les méthodes de Marsh et Cribier, a mis en évidence 19,54 mg d'arsenic dans les viscères du corps de Léon Besnard. On admet à l'époque que dans un cadavre frais, le poids moyen des viscères est de $6,5 \mathrm{~kg}$ (2). Cette dose supérieure à la normale (inférieure à $10 \mathrm{mg}$ ) prouverait, selon le rapport, une absorption d'arsenic avant sa mort. Il apparaît donc plausible de parler d'empoisonnement. Marie réfute les résultats de l'expert, mais un mandat de dépôt est signé et elle est accusée d'avoir empoisonné son mari. Elle part pour la Pierre Levée, la prison de Poitiers où elle entre le 21 juillet 1949, au soir. Inutile de décrire la nuit que va passer Marie en prison ! Sur les conseils de Maître Testard, avoué à Loudun ; Marie choisit pour sa défense maître Henri Cluzeau, inscrit au barreau de Poitiers et maître René Hayot, du barreau de Paris. Le $1^{\text {er }}$ Août 1949, les deux avocats de Marie demandent une contreexpertise qui leur est refusée tandis qu'on procède à l'exhumation des douze noms cités par Auguste Massip. Tous les prélèvements scellés sont expédiés à Marseille au laboratoire du docteur Béroud. Ce n'est qu'au moment des audiences qu'il sera fait état d'erreurs sur l'identité des cadavres prélevés, sur le nombre de bocaux expédiés et réceptionnés, sur des organes qui ne devaient pas exister et pour lesquels, figurent tout de même une analyse !

Le 31 octobre 1949, le docteur Béroud dépose son rapport. Il spécifie la présence d'arsenic dans les viscères de onze des douze cadavres, mais la terre et les morceaux de cercueil examinés ne renferment aucune trace d'arsenic (Tableau I) (2). Marie et ses avocats restent interloqués par ces résultats. En plus de l'homicide volontaire sur son mari, la voilà à présent inculpée d'homicide volontaire sur onze personnes !

Du 21 juillet 1949 au 19 février 1952, Marie subira des examens psychiatriques qui feront dire à l'un des experts que l'accusée est « anormalement normale » et responsable de ses actes. Ses avocats préparent la défense et travaillent avec des experts, Monsieur Perperot expert chimiste près la Cour d'appel de Paris et Georges Schuster, membre de l'Académie de Pharmacie qui interviendront comme témoins lors du procès. Maîtres Albert Gautrat et Jacqueline Favreau Colombier viennent renforcer la défense. La date du procès est fixée au 20 février 1952 devant la Cour d'assises de la Vienne à Poitiers. 
Tableau I : Récapitulatif des décès survenus dans l'entourage de Marie Besnard.

\begin{tabular}{|c|c|c|c|c|}
\hline $\mathbf{N}^{\circ}$ & Date décès & Age & Lien de parenté & Arsenic (mg)/viscères* \\
\hline 1 & $01 / 07 / 1927$ & 33 ans & $1^{\text {er époux de Marie }}$ & 60 \\
\hline 2 & $22 / 08 / 1938$ & 86 ans & Grand-tante de Marie & 35 \\
\hline 3 & $14 / 07 / 1939$ & 64 ans & Ami du couple Besnard & 18 \\
\hline 4 & $27 / 12 / 1941$ & 49 ans & Amie et veuve du précédent & 30 \\
\hline 5 & $14 / 05 / 1940$ & 78 ans & Père de Marie & 36 \\
\hline 6 & $02 / 09 / 1940$ & 92 ans & Grand-mère du 2 époux & très faible quantité \\
\hline 7 & $19 / 11 / 1940$ & 78 ans & Beau-père de Marie & 48 \\
\hline 8 & $16 / 01 / 1941$ & 68 ans & Belle-mère de Marie & 60 \\
\hline 9 & $27 / 03 / 1941$ & 45 ans & Belle-sœur de Marie & 30 \\
\hline 10 & $01 / 07 / 1945$ & 88 ans & Cousine du 2 époux & 48 \\
\hline 11 & $09 / 07 / 1945$ & 83 ans & Sœur de la précédente & 20 \\
\hline 12 & $14 / 01 / 1949$ & 71 ans & Mère de Marie & 48 \\
\hline
\end{tabular}

N.B : Second époux de Marie décédé le 25/10/1947, âgé de 55 ans : 19,54 mg d'As dans les viscères.

1 Dosages réalisés par le Dr Béroud (méthodes de Marsh-Cribier).

La terre et les morceaux de cercueil analysés ne contiennent pas d'arsenic.

* Dans un cadavre frais, le poids moyen des viscères est d'environ $6 \mathrm{~kg}$, réparti de la façon suivante

(2) : foie (1400 g) ; rate (150 g) ; reins (200 g); intestins (1100 g) ; estomac vide (100 g) ; estomac avec son contenu

(900 g) ; poumons (900 g); sang (150 g); cœur (300 g); sang du cœur (100 g) ; cerveau (1200 g).

\section{Le compte-rendu des trois procès (3).}

\section{Premier procès : Poitiers, 1952 (4)}

Trente mois après son arrestation, Marie Besnard est accusée de onze assassinats sur des parents et une amie. Les mobiles sont de deux ordres : recueil d'héritage et liaison adultérine. Le premier procès débute donc à Poitiers, le 20 février 1952. Après le tirage au sort des jurés, la cour présidée par le conseiller Edgar Favard, demande à ce que l'on fasse entrer l'accusée. Avant d'aborder l'affaire proprement dite Marie Besnard est accusée lors de la première journée d'avoir injustement encaissé un mandat postal destiné à sa tante et se voit condamnée à deux ans de prison et 12000 francs d'amende ! Le lendemain et les jours suivants, le président Favard questionne l'inculpée : tout son passé est étalé au grand jour avec en prime les insinuations formulées par les accusateurs. Il n'y a pas moins de 80 témoins à charge ! Le quatrième jour du procès sera marqué par l'intervention du docteur Béroud. Le Président évoque d'abord le problème des bocaux et de leur contenu et de toutes les négligences qui ont entouré les prélèvements et leur transport. Le docteur Béroud ne peut contredire ces constatations. On l'interroge ensuite sur les techniques employées pour détecter l'arsenic : il s'agit des méthodes de Marsh et Cribier mais ses réponses sont embrouillées et surtout, à un moment de l'audience, maître Gautrat lui présente six tubes préparés la nuit précédente par le docteur Roger Lebreton contenant tous un anneau d'antimoine (5). Le docteur Béroud ayant annoncé préalablement qu'il ne pouvait pas confondre arsenic et antimoine, reconnaît pourtant que trois des six tubes présentés renferment de l'arsenic. C'est la stupeur dans le prétoire : la renommée du docteur Béroud s'effondre, sa carrière aussi ! Le lendemain, il est absent pour cause de maladie. La défense le déplore, l'interrogatoire n'est pas terminé. Son collaborateur Aimé Médaille le remplace, mais ses réponses ne satisfont personne. On découvre des inexactitudes et des anomalies dans le rapport du docteur Béroud. Le 24 février les contre-experts cités par la défense démontrent que les analyses n'ont pas été menées avec assez de rigueur. Il y a des aberrations qui ne sont pas dignes d'un scientifique. Le docteur Béroud réapparaît à l'audience, soutenu par deux gendarmes; il refuse de débattre avec son confrère Schuster cité par les avocats de Marie Besnard. De son côté, le docteur Perperot déclare qu'il a retrouvé de l'arsenic dans la terre du cimetière de Loudun et par ailleurs si le rapport mentionne qu'un des empoisonnements a été réalisé par l'ingestion d'arséniate de plomb, il n'est pas fait mention de recherche de plomb dans les viscères.

Devant toutes ces erreurs, la défense demande une contre-expertise pour que la cour puisse juger Marie Besnard sur des rapports qui ne puissent pas engendrer de telles polémiques. Elle retourne en prison.

La seconde exhumation des victimes a lieu les 22 et 23 mars 1952. Les nouveaux experts, les professeurs Emile Kohn-Abrest, René Fabre et Henri Griffon, tous 
de Paris vont devoir employer les méthodes anciennes de Marsh et Cribier mais aussi une nouvelle technique américaine : la neutro-activation, applicable à l'analyse des cheveux (6). Le professeur René Piedelièvre, médecin légiste prélève les débris et restes cadavériques putréfiés ou disloqués, des copeaux de bois des cercueils, des restes de vêtements, de la terre ... bref tout ce que l'on a encore la chance de retrouver. 150 bocaux sont scellés et expédiés le jour même à Paris.

Durant deux années, divers rebondissements se succèdent et tiennent en suspens la date du second procès qui n'interviendra finalement qu'en mars 1954, à Bordeaux. De nombreuses questions se posent quant à la présence de l'arsenic dans la terre du cimetière, de sa solubilisation, de sa fixation éventuelle sur les vestiges cadavériques, notamment les cheveux, de la disparition possible d'arsenic au cours des phénomènes de putréfaction, de la surestimation des teneurs en arsenic retrouvées, du fait du dessèchement cadavérique et enfin de la migration possible de l'arsenic d'un cadavre à un autre cadavre par les eaux de ruissellement.

\section{Le second procès : Bordeaux, 1954 (7).}

Le second procès se déroule au palais de justice de Bordeaux du 15 mars au 1er avril 1954, sous la présidence de Henri Pourquery de Boisserin. L'avocat général annonce qu'il ne reste que six sujets dont la mort est déclarée suspecte sur les onze du dossier d'origine, puisque pour les autres soit il y avait prescription, soit les analyses ont mis en évidence des quantités trop faibles d'arsenic. Louise Pintou, les frères Massip et divers autres témoins se succèdent à la barre avant de laisser la place aux experts dès le 24 mars. Les professeurs Fabre, Kohn-Abrest et Griffon ont conclu à la même présence massive d'arsenic dans les cadavres examinés. Un rapport du professeur Frédéric JoliotCurie confirma la présence anormalement élevée d'arsenic dans ces mêmes cadavres. Le professeur Fabre avoue qu'il ne sait pas si l'arsenic est soluble dans un milieu qui se décompose. Le professeur Kohn-Abrest déclare que l'arsenic a été absorbé avant la mort mais ses résultats dans le cas de la mère de Marie Besnard montrent qu'elle aurait dû avaler $18 \mathrm{~g}$ de poison ce qui apparaît impossible à envisager. Les résultats du professeur Griffon sont contestés par la défense : les avocats insinuent qu'il y a accumulation d'incertitudes quant aux chiffres et aux modes de calcul employés. Un rapport du professeur Piedelièvre établi en 1954 confirma les conclusions des analyses de 1952 mais se montra plus nuancé que celui du docteur Béroud. Des « savants » choisis par Maître Hayot dont Marcel le Peintre, le docteur Ollivier et le professeur Keilling attestent que les eaux de circulation dissolvent l'arsenic qui réside dans les roches et qu'ainsi le corps de Léon Besnard submergé dans de l'eau contenant $0,1 \mathrm{mg} / \mathrm{l}$ d'arsenic s'est enrichi en ce métalloïde. Le docteur Ollivier démontre que la présence de microbes dans le sol fait passer l'arsenic de l'état insoluble à l'état soluble et qu'il peut ainsi imprégner le cadavre. Le professeur Keilling rappelle que la présence de zinc dans les cimetières est importante, que ce métal renferme de l'arsenic qui peut être ensuite solubilisé par voie microbienne et se fixer sur le cadavre. Et l'on apprend le lendemain que le jardinier du cimetière cultivait des pommes de terre et des céréales. Pour combattre les doryphores, l'arséniate de chaux faisait office d'insecticide!

L'arrivée d'un nouvel expert parisien, Louis Truffert, chargé de l'enseignement de la chimie toxicologique à la Faculté de Médecine de Paris, va arbitrer la bataille de l'arsenic. Pour lui l'arsenic est soluble, il peut être véhiculé dans les sols par les eaux de ruissellement ; il peut se fixer sur les cheveux et il résiste aux lavages les plus détergents du cheveu. Cette analyse remet en cause la certitude énoncée par les trois experts de la cour. Celle-ci ordonne alors de procéder à de nouvelles expertises. Les professeurs Maurice Lemoigne (membre de l'Académie des Sciences), René Truhaut (Faculté de Pharmacie de Paris) et Pierre Savel (Collège de France) sont chargés d'apporter un complément d'information et de mettre un terme à toutes les contradictions scientifiques. Maire Besnard est mise en liberté provisoire le 12 avril et retourne à Loudun le 12 mai 1954.

\section{Le troisième procès : Bordeaux, $1961(8)$.}

En 1955, l'équipe des experts est constituée. Le travail peut commencer. Il durera six ans. Le troisième procès débute le 20 novembre 1961 devant la Cour d'assises de Bordeaux. Marie entend pour la troisième fois la lecture de l'acte d'accusation qui n'a pas varié depuis 1952 et proteste toujours de son innocence. Le troisième jour s'ouvre sur les premiers débats des scientifiques. Maître Gautrat ne lâche toujours pas les experts. Il s'en prend au professeur Lemoigne à qui il reproche le manque de précision de ses contrôles, puis au professeur René Truhaut, au sujet des méthodes d'analyse utilisées. Le professeur Griffon est attaqué sur son analyse par radioactivation des cheveux de Léon Besnard. Vient ensuite l'analyse de la terre du cimetière de Loudun et là encore les quantités d'arsenic diffèrent selon les experts. Le zinc, les eaux de ruissellement sont autant d'incertitudes quant à leur implication dans le transport de l'arsenic vers les tombes. Des erreurs de quantité, de virgule achèvent de semer le trouble chez les jurés. Plus grave encore, sur les fiches de laboratoire du professeur Griffon, on note des ratures, des résultats différents de ceux du rapport final et certaines fiches ont été détruites. Louis Truffert est en désaccord avec les experts sur l'interprétation de leurs résultats et estime que la présence d'arsenic dans les corps des victimes est d'origine externe liée à l'imprégnation par les eaux de ruissellement, hypothèse qui sera corroborée par le docteur Ollivier et le professeur Keilling. Bref, au fur et à mesure que les jours passent, les expertises 
officielles sur lesquelles se fondaient les juges pour prononcer un verdict s'effondrent. Il ne reste plus rien à l'accusation si ce n'est les ragots de Loudun.

Suivent les plaidoiries de la partie civile, du procureur général et de la défense et enfin le verdict des jurés qui aux questions portant sur la culpabilité de Marie Davaillaud, veuve Besnard, dans la mort des onze victimes supposées empoisonnées, répond par la négative. En conséquence de quoi Marie Davaillaud, épouse Besnard est acquittée des fins de la poursuite.

\section{Réflexions et conclusion.}

Sur le plan médiatique l'affaire Marie Besnard demeure une affaire bien troublante. Certes les experts requis par les autorités judiciaires ne se sont pas totalement investis dans leur mission, ils n'ont pas été assez rigoureux sur le plan des analyses et de leur interprétation et surtout ils ont ignoré en grande partie la procédure judiciaire. Par contre, le travail de la défense a été considérable, mais elle a détourné, en quelque sorte la mission des experts toxicologues vers des problèmes ou des situations qui ne relevaient pas de leur compétence. Ils étaient là pour infirmer ou confirmer un empoisonnement arsenical sur la base de leurs connaissances ou de leur expérience à savoir si la quantité d'arsenic contenu dans les organes analysés était ou non supérieure à la normale. Or ils sont tous à peu près d'accord : chez onze cadavres sur douze, ils retrouvent des quantités d'arsenic supérieures à la normale et en tout cas supérieures à celles des cadavres témoins non concernés par cette affaire et qui ont pourtant séjourné dans le même cimetière. On a demandé à ces experts d'interpréter des données environnementales sur lesquelles ils n'avaient aucune ou très peu d'idées : la migration de l'arsenic dans le sol, sa solubilisation dans l'eau et sa fixation possible sur les vestiges cadavériques.

Tout au long de ces procès, on a l'impression que l'inculpée attend la démonstration de l'empoisonnement et que celle-ci n'arrive pas à se faire jour car la défense estime pour sa part que l'arsenic présent dans les prélèvements cadavériques ne peut provenir que de l'environnement. Évidemment elle est aidée dans son travail par le manque de rigueur des experts qui pour la plupart n'ont pas assez prêté attention au recueil des prélèvements, aux analyses qu'ils auraient dû pratiquer euxmêmes au lieu de les confier à leurs adjoints et enfin à l'interprétation des résultats qu'ils auraient du soumettre à l'avis d'autres experts au lieu de tenir compte de leur seule compétence en la matière.

C'est un peu un dialogue de sourds ou une querelle entre une accusation persuadée de la culpabilité de la présumée coupable qui accepte les données des experts requis par la justice et une défense qui contre-attaque chaque point soulevé, émet un doute sur chaque résul- tat et déstabilise des experts renommés dans un domaine bien spécifique sur des sujets qu'ils ne maîtrisent pas et pour lesquels évidemment leurs réponses sont peu convaincantes.

L'affaire Marie Besnard démontre que la toute puissance donnée jusqu'alors aux experts peut être démantelée. Selon Maître Hayot « il faut contredire l'expertise de front et ne jamais laisser les experts débattre uniquement entre eux ». Nous osons avouer qu'au cours de notre vie d'expert, nous avons ressenti dans le prétoire cette sorte d'invincibilité liée à la certitude de la véracité de nos résultats d'analyse et à notre foi dans les connaissances scientifiques que nous possédions face à une institution totalement étrangère à cette culture. Mais, à qui la faute ? À la justice qui ne veut pas essayer de comprendre nos problèmes analytiques ou à nous qui n'essayons pas de les leur présenter sous un jour plus simple? Il est vrai qu'il existe aujourd'hui des contrôles de qualité et que ces contrôles permettent aux experts de confronter leurs techniques et de s'assurer de la validité de leurs résultats, cependant il restera toujours un problème d'envergure, celui de l'interprétation de ces mêmes résultats. Alors quelles leçons tirer aujourd'hui de cette affaire ? En premier lieu l'expert doit surveiller de très près l'analyse qui lui a été confiée. S'il ne peut l'exécuter lui-même, il a toujours la possibilité de faire nommer un sapiteur. Enfin, lors de l'interprétation des résultats, il doit toujours préserver une part de scepticisme, d'humilité intellectuelle, de lucidité et devrait toujours se remettre en question. En effet, la toxicologie comme toute science, progresse d'erreur dépassée en erreur dépassée. C'est ce que l'on appelle le progrès scientifique rythmé par l'évolution des connaissances. L'expert n'est donc pas un professionnel de la vérité, mais il doit être un professionnel de la connaissance au service de la justice pour dire le possiblement vrai et le certainement faux. C'est à ce prix qu'il sera reconnu.

\section{Références}

1. Mamouni S. L'affaire Besnard. De Vecchi S.A., Paris, 1999, $134 \mathrm{p}$.

2. Ducasse-Pouvereau S. Les expertises toxicologiques lors de l'affaire Marie Besnard. Th. Doct. Pharm. Bordeaux ; $1987, \mathrm{~N}^{\circ} 84,136 \mathrm{p}$.

3. Favreau-Colombier J. Marie Besnard, le procès du siècle. Ed. Privat, Toulouse, 1999, 448 p.

4. Paris Match $\mathrm{N}^{\circ} 155$ du $1^{\text {er }}$ au 7 mars 1952.

5. Le Breton R., Garrat J. Interdit de se tromper : 40 ans d'expertises médico-légales. Ed. Plon, Paris, 1993

6. Castagnou R., Argilas-Larcebeau S., Bonini M. Cours de Toxicologie, 1980, Tome 2, p 190.

7. Paris Match $\mathrm{N}^{\circ} 260$ du 20 au 27 mars 1954.

8. Paris Match $\mathrm{N}^{\circ} 662$, numéro spécial Noël 1961. 